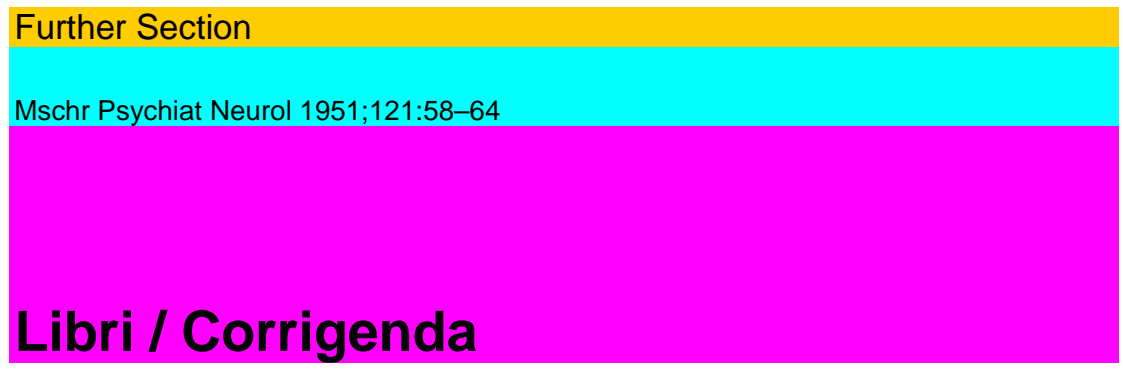


$D$, Bovet et F. Bovet-Nitti: Structure et activit6 pharmacodynamique des m6dica-ments du Systeme nerveux veg\&atif. Verlag S. Karger AG., Basel, 1948, VIII et 832 pages avec 32 flg., Fr. 85.-.

Die Verfasser behandeln in den großen Abschnitten Adrenalin, Acetylcholin und Histamin, alles was in pharmakologischer und therapeutischer Hinsicht zum vegetativen System Bezug hat. Der neueste Stand der Forschung auf den therapeutisch hochinteressanten Gebieten der AntiMstarninica, Adrenolytica und Sym-patbicolytica, der anticholinergischen Stoffe, der nicotin- und der curareartig wirkenden Pharmaca ist ausführlich dargestellt, wobei nicht nur die synthetischen, sondern auch die Wirkstoffe pflanzlicher Herkunft gebührende Berücksichtigung finden.

Das Buch gewinnt ganz besonderen Wert durch die zahlreichen Originalarbeiten, die beide Verfasser selbst zu diesen Themata geliefert haben, und durch die gewissenhafte Berücksichtigung der außerordentlich umfangreichen Literatur. Es entstand dabei ein Handbuch, das dem im gleichen Verlag erschienenen Standard-

Libri

59

werk von Guggenheim über die biogenen Amine als glückliche Ergänzung an die Seite zu stellen ist. Die Entwicklung der chemisch-pharmazeutischen Forschung ist besonders berücksichtigt, so daß der Bovet wohl zu einem Handbuch werden wird für alle Chemiker und Pharmakologen, die sich mit diesem Gebiet befassen; darüber hinaus ist er auch dem an chemisch-pharmazeutischen Fragen interessierten Arzt und Biologen ein zuverlässiges Nachschlagwerk.

R. Domenjoz, Basel.

Ernst Speer: Vom Wesen der Neurose und von ihren Erscheinungsformen. Georg Thieme Verlag Stuttgart, 1949. 2. Auflage. 106 S. DM 7.80.

In dem kleinen Buch, das erstmals 1938 erschien, stellt der Verfasser das Wesen der Neurose aus seiner praktischen Erfahrung dar. Neurose ist danach ganz allgemein Störung der Erlebnisverarbeitung. In dem ersten Teil, der allgemeine Gesichtspunkte behandelt, wird das Hauptgewicht auf die durch die Neurose bewirkte Absonderung von der Gemeinschaft gelegt. Sie wird für die „Verfemung" (eine Art Abwehr gegen diese Absonderung) und das „Leid der Neurose" letztlich verantwortlich gemacht. Eine spezifische Schuld des Kranken wird verneint. Ferner schreibt Speer der Neurose einen Erholungswert zu, indem er sie in Analogie zu dem Schlaf stellt, der übermüdete Individuen wieder ,gemeinschaftsfähig" macht. In ähnlicher Weise bewirkt die Zwischenlösung des neurotischen Symptoms vorübergehende Entspannung in der Ausnahmesituation. In einem geschichtlichen Rückblick wird „das Wechselspiel der Kräfte", die im Individuum zur Erhaltung seiner selbst und zur Gewährleistung der Gemeinschaft wirken, aufgezeigt, wobei es nicht ohne Seitenhiebe auf Philosophen und Theologen abgeht, die ohne praktische Erfahrung moralisierend Wesen der Geisteskrankheit und Neurose verkannt hätten. In einem zweiten Teil werden die Erscheinungsformen der Neurose besprochen, ohne daß eine Systematik gegeben wird, die nach der Meinung des Verfassers wegen der unendlichen Vielfalt neurotischer Manifestationen verfehlt wäre. Um „bildhafte Vorstellungen vom Wesen der Neurose zu vermitteln", werden in bunter Folge Zwangsneurose, Sucht, Reifungskrisen und die sogenannten Organneurosen anhand von charakteristischen Beispielen dargestellt. Auch dabei verzichtet Speer auf theoretische Konstruktionen und räumliche Bilder für Bewußtes und Unbewußtes.

Eine unheilvolle Rolle spielt in dem Buch der Begriff „Entartung", der nicht klar von Krankheit oder Psychopathie abgegrenzt und nur indirekt zur Konstitution in Beziehung gebracht wird durch Nachweis des Familienbildes „entarteter" Familien. Entartung bedeutet nach dem Verfasser bald etwas Wertnegatives im Sinne der Gemeinschaftsunfähigkeit („das abscheuliche Bild der schwer Entarteten"; Haltlose, Unerziehbare etc.), bald Wertpositives, denn es gibt auch „hochbegabte, wertvolle Entartete" (S. 106). Echte Neurose wird dem „neurotischen Unwesen Entarteter" gegenübergestellt. Schließlich wird die Reihe: Schizo-thym-Schizoid-Schizophren als 
die Entartungslinie der menschlichen Persönlichkeit schlechthin bezeichnet! Diese undurchsichtige Anwendung von „Entartung" und die Uberbetonung der Gemeinschaft wirft ein schiefes Licht auf das Buch. Der Autor mißt nämlich den Wert einer Persönlichkeit nur nach ihrem Wert für die Gemeinschaft. S. 31 steht sogar folgende Fußnote: „Sünde kommt von Sondern. Die Theo-

60

Libri

logen sagen, daß Sünde Absonderung von Gott sei. Sie vergessen aber zu sagen, daß dieser Gott letzten Endes eben die Gemeinschaft ist mit all ihrem überpersönlichen Recht."

Über solche Behauptungen ist man füglich erstaunt, wird man doch dadurch unwillkürlich an das Jahr der ersten Ausgabe erinnert, wo im Namen des „überpersönlichen Rechtes" einer Volksgemeinschaft „Entarteten" die Lebensberechtigung abgesprochen wurde. Daß dabei Wissenschafter und nicht Philosophen oder Theologen am Werke waren, ist uns wenistens noch in Erinnerung! Ähnlich berührt, daß Sigmund Freud nur beiläufig in negativem Sinne zitiert wird und schließlich der ernstgemeinte Vorschlag, dem Staate die Ehevermittlung aufzutragen, weil man damit durch „amtliche Gesundheitszeugnisse erbbiologisch ungünstigen Mischungen vorbeugen" und die charakterliche Eignung der Partner „unter Beiziehung bester graphologischer Gutachten" prüfen könnte! Man möchte wünschen, daß nicht nur der Begriff der „Entartung" aus der wissenschaftlichen Literatur, sondern auch die Bevormundung durch den Beamtenstaat verschwinden.

Hans Heimann (Waldau).

Gottfried Ewald: Lehrbuch der Neurologie und Psychiatrie.

Urban und Schwarzenberg, Berlin/München 1948. 2. Aufl. 478 S., 32 DM.

Der ersten Auflage dieses Buches, die seinerzeit in der Monatsschrift angezeigtwurde, ist rasch ein zweite gefolgt, und dies spricht nicht nur dafür, daß das Bedürfnis nach Lehrbüchern im heutigen Deutschland groß ist, sondern es spricht auchfür die Vorzüglichkeit dieses Werkes. Meines Wissens ist es das einzige Lehrbuch,das auf knappem Raum von etwa 500 Seiten sowohl die Nervenkrankheiten, wiedie psychischen Erkrankungen darstellt. Dazu kommt noch, daß jedem der beidenTeile eine allgemeine theoretische Einführung vorausgeht, sowohl über die Grundzüge der Anatomie und der Physiologie des Nervensystems, wie auch über diePsychopathologie, die unter dem Titel, „Aufbau der Person", originell und anschaulich beschrieben wird. Sogar ein Überblick über die gerichtliche Psychiatriefehlt zum Schlüsse nicht. Gegenüber der ersten Auflage mußte diesmal über dasverblichene Sterilisationsgesetz nicht mehr gesprochen werden, und damit war esmöglich die Erbbiologie überhaupt etwas kürzer zu behandeln. Im Übrigen warenwenig Änderungen gegenüber der ersten, im Krieg erschienenen Auflage notwendig.Die Darstellung ist knapp und lebendig, lesbar und begrifflich scharf und hebtgeschickt das Wesentliche heraus. Daß neben den abnormen Reaktionen die Neurosen im engeren Sinne etwas zu kurz kommen, sei nur angemerkt. Kurz, das Lehrbuch Ewalds dürfte auch in der zweiten Auflage dankbare Leser und Schülerfinden, und es verdient weite Verbreitung.

Wyrsch.

Viktor E. Frankl: Der unbedingte Mensch. Verlagsbuchhandlung Franz Deuticke, Wien 1949.

In dem als „metaklinische Vorlesungen" bezeichneten Buch führt der Verfasser klar und fesselnd in eine Metaphysik der Medizin ein. In ihr sind zeitgenössische philosophische Richtungen, gekennzeichnet durch die Namen: Heidegger, Jaspers, Nikolai Hartmann, L. Binswanger u. a., selbständig verarbeitet. Es geht Frankl darum, metaphysische Probleme, die dem Arzt und Forscher tag-

Libri

61

täglich begegnen, aufzuwerfen und von einem ontologischen Standpunkt aus zu diskutieren. Es soll keine „Systematik" sondern eine „Problematik" der Metaphysik dargestellt werden, die zu eigener Stellungnahme herausfordert und an existentielle Entscheidung appelliert. Es ist dies dem Verfasser in dem vorliegenden Werk ohne 
Zweifel gelungen. Kein Leser, dem die Frage nach dem Menschsein nahegeht und den die Enge der monistischen Weltbilder unbefriedigt läßt, wird es ohne Ergriffenheit zu Ende lesen, selbst wenn man mancherorts anderer Meinung ist und oft widersprechen möchte. Es werden in einzelnen Kapiteln besprochen: Leib-Seeleproblem, Problem des Geistes als Wesen und Werden des Geistes (veranschaulicht an Hirnpathologie und Phylogenese, an Ontogenese und Erbpathologie), das Problem der Sterblichkeit, das Problem der Willensfreiheit. Ohne auf Einzelheiten einzugehen, was wegen des beschränkten Raumes nicht möglich ist, sei aus dem Inhalt folgendes zusammengefaßt: Frankl geht von der Seinsauffassung N. Hartmanns aus, wonach das Sein in vier Schichten ,zerfällt": In diejenige des Physikalischen, die des Organischen, die des Seelischen und die des Geistigen. Die Trennung der Schichten ist für Frankl jedoch nur ein „heuristisches Prinzip", nicht ein reales Zerfallen, als ob der Mensch etwa aus diesen vier Teilen zusammengesetzt wäre. Frankl gibt dem Geistigen das Primat, wobei er „Geist" als „geistige Person" der „Existenz" gleichsetzt. Die andern Seinsschichten können „Geist" nur bedingen, nicht bewirken. Die „geistige Person" ist deshalb frei. Das mehrfach angeführte Zitat von N. Hartmann gibt diese Verhältnisse in prägnanter Form wieder: „Autonomie trotz Dependenz". Frankl führt aus: „Überall ist Spielraum, der ausgespart bleibt von Bedingungen, ausgefüllt vom Unbedingten!" Von hier aus wird die Würde des Menschen abgeleitet, die auch in Geisteskranken gewahrt bleibt, weil hier die „geistige Person" zwar verschüttet, verdeckt und ohnmächtig ist, jedoch nie selbst von der Krankheit mitbetroffen oder gar zerstört. Es ist dies ein „Credo", das Frankl zur Voraussetzung jeder psychiatrischen Tätigkeit macht, weil Therapie nur sinnvoll sein kann, wenn sie Hilfe für die „geistige Person" ist, im besondern Falle Hilfe gegen den „Einbruch aus dem Psychosomatischen" der Geisteskrankheit. Frankl postuliert ferner einen „noo-psychischen Antagonismus" im Gegensatz zu dem psychophysischen Parallelismus. Dabei ist festzuhalten, daß der noo-psychische Gegensatz, Gegensatz von Geist und Seele, nicht mit der falschen Akzentuierung Klages" (zersetzende Macht des Geistes) sondern als Übergeordnetsein des Geistes aufgefaßt wird. Der „Geist" macht sich das „Psychophysikum" Untertan. Es muß betont werden, daß Frankl unter Geist immer ,geistige Person" (Existenz) versteht und nicht bloß den Raum der objektiven Gehalte des Seelischen (Jaspers). In diesem noopsychischen Gegensatz sieht Frankl die einzigartige Begründung seiner existenzanalytischen Betrachtungsweise und letztlich dessen, was er „Logotherapie" genannt hat. Er versteht unter letzterem ein Erwecken und Appellieren, die sich an die ,geistige Person" des Kranken wenden, um den durch die übliche Therapie nicht angehbaren Rest der Krankheit, sei es Neurose, Psychose oder auch Charakterdefekt, wo nicht zu überwinden, so doch zu isolieren, und damit das Individuum zu „befreien". Es gilt also, die „richtige" Einstellung zu seinem Leiden zu finden. Frankl unterscheidet vier Einstellungsmögblchkeiten des Kranken zu seiner Krankheit: 1) schlechte Passivität: Blindes Gehorchen gegenüber triebhaften Impulsen, oder Selbstvergessenheit in neurotischem Fatalismus. Dabei ist hervorzuheben, wie sehr sich Frankl damit von den Thesen Freuds distanziert,

62

Libri

dem er geradezu vorwirft, den Fatalismus des „Nicht-anders-Könnens" gezüchtet zu haben, eine Kritik, wie sie unter anderm Vertreter des christliehen Bekenntnisses von jeher gegen die Auffassung des Menschen durch die Psychoanalytiker eingenommen haben. Ein weiter Weg von der psychoanalytischen Behandlung Freuds und seiner Schüler zur „Logotherapie" Frankls! 2) Die schlechte Aktivität: das noch intensivere und unermüdliche Ankämpfen gegen die Symptome. 3) Die rechte Aktivität: Objektivieren der Restsymptome und dadurch Distanzgewinnen. 4) Die rechte Passivität: Erziehung an dem schicksalshaften Kern der Krankheit vorbeizuleben, ihn zu ignorieren.

Es mindert den Wert des Buches keineswegs, wenn man beim Lesen sehr oft mit Frankl nicht einig geht. Ohne ausführliche Kritik seien doch folgende zwei Punkte hervorgehoben: 1) Der „geistigen Person" des Arztes gehört es zu, über die Dinge hinaus den metaphysischen Grund zu suchen, empfängt er doch von seiner Auffassung des Menschseins Kraft und Lenkung seines Wirkens. Wohl ist die konkrete Auseinandersetzung und Stellungnahme zu diesen jederzeit auftauchenden Fragen als die vielgerühmte frag- und gedankenlose Voraussetzungs-losigkeit, die eine unbewußte metaphische Vorwegnahme par excellence darstellt. Ob man jedoch von der 
metaphysischen Ebene so rasch zur Empirie wechseln darf, wie es Frankl bei der beiläufig gegebenen Deutung des „Dejä vu" tut, das er als „vorübergehenden Ausfall der Zeitigung des Daseins" deutet, also empirische Tatbestände in metaphysisches Gewand kleidet, ist uns sehr fragwürdig. Wir möchten in diesem Zusammenhang auf die scharfe Trennung des empirischpsychologischen und des metaphysischen Verstehens hinweisen, wie sie Jaspers entwickelt (allgemeine Psychopathologie). Metaphysische Spekulationen nicht als Grund, sondern als Erklärungsversuche innerhalb der Forschung machen die empirischen Tatbestände nicht „durchsichtig" und verfälschen den Sinn metaphysischer Erhellung. Sie fordern zudem zu einer berechtigten Reaktion heraus. Es ist Sache des Philosophierenden, die Bausteine der Forschung als „Chiffre" metaphysisch zu interpretieren.

2) Die „Logotherapie" Frankls, als Appellieren an das geistige Selbst des Kranken in existentieller Kommunikation zwischen Arzt und Patient, ist keine eigentliche medizinische Therapie, weil die wesentlichste Voraussetzung, die „Kommunikation", durch den Therapeuten nicht erzeugbar ist. Arzt und Patient müssen sie beide in radikalem Offensein miteinander suchen. Letztlich aber ist sie existentiell in Transzendenz gegründet. Es verhält sich mit der Logotherapie wie mit der Seelsorge. Man könnte für sie kein Honorar verlangen! Damit, daß man jemandem rät, sich von seinen Symptomen zu distanzieren und sein Schicksal zu tragen (rechte Aktivität und rechte Passivität Frankls), ist nicht ärztliche Hilfe geleistet, sondern menschliche, brüderliche in Kommunikation. Das will nun nicht besagen, daß der Arzt diese Hilfe nicht leisten kann und von jeher (auch ohne den schönen Namen) geleistet hat, weil wirksame und lebensnahe Psychotherapie letzten Endes über die rein ärztliche Kunst hinausführt zu brüderlicher Hilfe.

Hans Heimann, Waldau.

Hans Jacob: Der Erlebniswandel bei Späterblindeten. Abhandlungen zur Psychiatrie, Psychologie, Psychopathologie und Grenzgebieten. Herausgegeben von Prof. Dr. med. Hans Bürger-Prinz, Hamburg. H. H. Nölke Verlag, Hamburg.

Libri

63

Die als „Beitrag zur Psychopathologie der optischen Wahrnehmung" vorliegende Arbeit stützt sich auf sorgfältigste Untersuchungen und auf Selbstschilderungen Kriegsverletzter des zweiten oder dritten Lebensjahrzehnts, die akut traumatisch total erblindeten infolge peripherer Verletzungen (Bulbus- oder Sehnervenzerstörung). Die zahlreichen bemerkenswerten Ergebnisse werden nach phänomenologischen Gesichtspunkten analysiert, ausführlich dargestellt und schließlich in das Gesamt des Wahrnehmungsaktes eingeordnet. $\mathrm{Zu}$ der einschlägigen Literatur wird Stellung bezogen. Aus der eindrucksvollen Arbeit, die jedem, der sich für die subjektiv erlebten Phänomene der Wahrnehmungsfunktion, nicht nur für deren Leistung, interessiert, bestens empfohlen werden kann, sei folgendes kurz zusammengefaßt:

Sehverlust nach peripherer Späterblindung ist mehr als Ausfall der optischen Wahrnehmungswelt, die durch gesteigerte Hinwendung auf außeroptische Wahrnehmungsmöglichkeiten mehr oder weniger kompensiert wird. Tätiges Ergreifen der Umwelt durch den Erblindeten mit Hilfe außeroptischer Wahrnehmung und Visualisieren ergibt sich nicht primär, sondern erst mittelbar als menschliche Leistung innerhalb eines gewandelten „Subjekt-Umweltbezuges". Das Sehen als Akt der Wahrnehmung, als gewphnte Form der Hinwendung, das durch ständige Bezugnahme zur gewohnten optischen Umwelt beeindruckt, führt und von der Hinwendung auf sich selbst ablenkt, fällt aus. Es kommt zu einer tiefgreifenden „Umschichtung im vitalen Persönlichkeitsbereich", die erst nach „katastrophenreichem Übergangsstadium" mit mannigfaltigen optischen Täuschungserlebnissen (Fotismen, szenisch-optischen Erscheinungen etc.) schließlich eine Adaptation erlaubt. Mit dem Einsetzen der Erblindung ist das Kontinuurn des optischen Erlebens gewährleistet durch die schlagartig „einschnappende, initiale Umweltschwärze", die den Charakter einer leibhaftigen Bewußtheit \{Jaspers) hat. Nach Tagen oder Wochen wird sie von dem frontalen „Graunebelblickfeld" (Surrogatblickfeld) abgelöst, das dem Gesichtsfeld des Sehgesunden entspricht. Das initiale optische „Ringsumer-leben" wandelt sich damit zni einem „Vorsichsehen", das erst die gewohnte sinnliche Einheit eigenkörperlicher Raumeinordnung (vorn-hinten etc.) ermöglicht. Durch Ausbleiben des gewohnten Blickfeldwandels in Abhängigkeit von Eigenbewegungen entsteht eine erlebbare 
Körperschemastörung. Durch Verlust der Möglichkeit, mit sehendem Eingreifen, bzw. Vorwegnehmen und Übernehmen außeroptischer Umweltsreize den außeroptischen Wahrnehmungsapparat zu entlasten, ergibt sich hier eine Reizüberlastung und eine immer gegenwärtige Reizerwartungssituation, die eine gesteigerte Hinwendung auf verändertes körperliches Erleben bewirkt. In der neuentstehenden optischen Surrogatwelt, die eigenartigerweise im Vordergrund des Erlebens des Blinden steht, repräsentiert sich eigenkörperliches Erleben, Stimmung, körperliches und seelisches Versagen in Helligkeitswandel des Graunebelblickfeldes und Stärke des Visualisierungsvermögens. Es taucht ferner das vom Sehgesunden „Übersehene" auf: Begrenzung des Gesichtsfeldes durch Orbitaband und Nase, im Blickfeld erscheinende Gliedbewegungen, alles in allem RärEai.iJmkelirung der „negativen Leistung" ( $v$. Weizsäcker) im optischen Erleben, einer Leistung, die durch „Übersehen" erst ein erfolgreiches Handeln ermöglicht. In der Surrogatwelt ist sogar das vom Sehgesunden übersehene Moment der Einschränkung im optischen Wahrnehmungsakt (prädikativer Akt von v. Weizsäcker) optisch gegeben, das optische Teüstück wird vom Kranken mit sinnlicher Deutlichkeit erlebt. Schließlich tauchen optische Begebenheiten auf, die mit dem Gefühl

64

Libri

der Selbstentfremdung erlebt werden, was nach dem Verfasser am deutlichsten auf die Erschütterung der vitalen Person hindeutet. In den Endstadien besteht diese Erschütterung noch latent weiter und macht sich gelegentlich in Versagungszu-ständen als „vision nulle" (Dufour) bemerkbar. Der Vergleich mit einem Fall Pötzls von cerebraler, traumatischer Amaurose zeigt, daß bei Zerstörung der Sehrinde, derselbe optische Erlebniswandel eintritt wie bei peripheren Späterblindeten. Die erlebten Phänomene des Erblindeten werden schließlich in Beziehung zu phänomenologisch verwandten optischen Gegebenheiten in vitalen Zustandsänderungen (Einschlaf-, Wacherlebnisse) und Ausnahmezuständen (Erschöpfung, Mescalin) Sehgesunder gebracht und an der Übereinstimmung der Phänomene festgestellt, „daß das Ausblenden des optischen Sinnensektors grundsätzlich — hinsichtlich der Erschütterung im Bereiche vitaler Schichten, aber auch der wahrnehmenden und handelnden Umweltkommunikation, soweit sie sich in der Sphäre des Optischen verwirklicht - ein in den Grundzügen sehr ähnliches Geschehen in Gang setzt, wie die Schädigung des menschlichen Organismus in Ausnahmezuständen". Der Verfasser sieht darin eine Stütze der Auffassung von Beringer, Bürger-Prinz, Mayer-Gross und Stein," daß für das Gesamterleben, insbesondere für den Wandel optischer Wahrnehmungen, vor allem im Mescalinrausch, aber auch im Delir Funktionsstörungen des Sinnesapparates notwendige Voraussetzungen darstellen."

Hans Heimann, Waldau.

\section{Excerpta Medica}

Section VIII: Neurology and Psychiatry. Board of Chief Editors: M. W. Woerdeman, M. D., Chairman, Amsterdam; M. Fischbein. M. D., Chicago; W. P. Zeeman, M. D., Amsterdam. Subskriptionspreis $£ 5$ sl2;Dfl. 60.-oder Fr.106.—. Jährlich ein Band von etwa 1000 Seiten.

Seit zwei Jahren erscheinen in Amsterdam, in 15 Sektionen untergeteilt, alsgemeinnütziges Unternehmen die Zeitschriftenreihen der Excerpta Medica. Dasganze, international großzügig organisiert, stellt ein die gesamte Medizin umfassendesmonatlich fortlaufend erscheinendes Referatenwerk in englischer Sprache dar.Sektion VIII ist dem Gebiet der Neurologie und Psychiatrie in allen ihren Zweigengewidmet. Die beiden jetzt vollständig vorliegenden Jahrgänge lassen erkennen,daß die Berichterstattung rasch und so vollständig wie möglich erfolgt. Einteilungund Register sind übersichtlich und gut, so daß jeder Interessent, und dies sollteneigentlich alle Neurologen und Psychiater sein, sich schnell über die Neuerscheinungen in allen Sondergebieten orientieren kann. Die Zeitschrift ist angelegentlichst zu empfehlen.

E. Grünthal. 


\section{Corrigenda}

I. In der Arbeit von E. Grünthal „Beiträge zur Kenntnis des extrapyramidal-motorischen Systems" (Mschr. f. Psych

u. Neurol. 120

337

1950) ist die Num-merierung der Abbildungslegenden versehentlich verwechselt worden. Es sollte heißen: Abb. 4 statt Abb. 2

Abb. 2 statt Abb. 3 und Abb. 3 statt Abb. 4. 